\title{
GENETIC DIVERSITY IN WHEAT GENOTYPES USING SIMPLE SEQUENCE REPEAT (SSR) MARKERS
}

\author{
El-Rawy, M. A.; A. S. Taghian; H. M. El-Aref; B. E. Abd El-fatah and S. A. El-Sanousy
}

Genetic Department, Faculty of Agriculture, Assiut University, Egypt

\begin{abstract}
A total of 73 genotypes including 65 hexaploid (Triticum aestivum L.) and 8 tetraploid (Triticum durum L) wheat varieties were used in this study. Eleven microsatellite markers were used to test the genetic diversity of wheat genotypes, yielding a polymorphism. The total number of detected alleles was 89 with an average allele number of 8.1 per locus. The maximum number of polymorphic alleles ( 9 bands) was obtained by the primer SSR- 9 while the minimum number ( 1 allele) was recorded for primer SSR-11 with an average 5 alleles per primer. Polymorphism percentage ranged from as low as $50 \%$ (SSR-1, SSR-2 and SSR-5) to as high as 100\% (SSR-10 and SSR-11) with an average of $68.24 \%$. Eleven alleles at molecular size [219 bp and 188 bp (SSR-1), 239 bp (SSR-3), 635 bp and 503 bp (SSR4), $358 \mathrm{bp}$ and $331 \mathrm{bp}$ (SSR-8), $250 \mathrm{bp}, 226 \mathrm{bp}$ and $210 \mathrm{bp}$ (SSR-9) and $532 \mathrm{bp}$ (SSR-11)], were present only in bread wheat genotypes, while one allele at molecular size 312 bp generated with primer (SSR6) was unique to durum wheat genotypes. These alleles could be used as marker to distinguish the durum wheat from the bread wheat genotypes. Polymorphism information content (PIC) value ranged from 0.06 (primer SSR-6) to 0.2 (primer SSR-11) with an average value of 0.13 . These results reflect the wide range between the frequencies of alleles at loci of SSR studied in the tested wheat varieties. The dendrogram grouped the 73 wheat genotypes according to their ploidy levels into two main clusters. Cluster 1 included the durum wheat genotypes, while cluster 2 contained all bread wheat genotypes.
\end{abstract}

Keywords: durum wheat, bread wheat, SSR markers, wheat genotypes

\section{INTRODUCTION}

Wheat is an important cereal crop used as a major human consumable commodity in most areas of the world. The species of Triticum are grouped into diploids $(2 n=2 x=14)$, tetraploids $(2 n=4 x=28)$ and hexaploids $(2 n=6 x=42)$. Triticum aestivum, common bread wheat, contains 3 different but genetically related genomes ( $A, B$ and $D$ ) with a total genomic size of $1.7 \times 10^{10}$ base pairs, illustrating the complex nature of wheat genome. Durum wheat or macaroni wheat (Triticum durum or Triticum turgidum subsp. durum) is the only tetraploid species of wheat of commercial importance that is widely cultivated today. It is an allotetraploid species with $2 n=4 x=28$ ( $A A B B$ genome) that originated through intergeneric hybridization and polyploidization involving two diploid grass species $T$. urartu $(2 n=2 x=14, A A$ genome $)$ and a B-genome that is diploid related to Aegilops speltoides ( $2 \mathrm{n}=2 \mathrm{x}=14$, BB genome) (Kihara 1944 and McFadden and Sears 1946).

Several molecular markers like random amplified polymorphic DNAs (RAPD), inter simple sequence repeats (ISSR) and simple sequence repeats (SSRs) are presently available to assess the variability and diversity at molecular level (Palombi and Damiano, 2002). Hence, keeping in view the importance of these aspects the present study has been planned. This is considered as the most effective method for qualifying the degree of genetic diversity among the genotypes included in the study.

Genetic diversity is one of the key factors for improvement many crop plants including wheat. Plant breeders rely on the availability of genetic diversity during selection in cultivar development. The efficiency of genetic gain by

Special volume for the first International Conference of Genetic Engineering and Biotechnology, 
selection can be improved if the patterns of genetic diversity within a population of breeding lines are

known. Genetic similarity and or distance estimates among genotypes are helpful in the selection of parents to be used in a breeding program (Van-Becelaere et al., 2005). Genetic diversity can be assessed from pedigree analysis, morphological traits or using molecular markers (Pejic et al., 1998). However, diversity estimates based on pedigree analysis have generally been found inflated and unrealistic (Fufa et al., 2005). Genetic diversity estimates based on morphological traits, on the other hand, suffer from the drawback that such traits are limited in number and are influenced by the environment (Maric et al., 2004).

Molecular markers are useful tools for estimating genetic diversity as these are not influenced by environment and do not require previous pedigree information. Among the molecular markers techniques, random amplified polymorphic DNA (RAPD) which introduced by Williams et al. (1990).

DNA markers are technology that can increase breeding progress, especially for traits that are difficult to select under field conditions and that are controlled by multiple genes. Microsatellites are repeating sequences of 2-6 base pairs of DNA (SSRs; Simple sequence repeats) and are among the most stable markers of genetic variation and divergence among wheat genotypes because they are multiallelic, chromosome-specific and evenly distributed along chromosomes. Microsatellite genotyping is used for genetic biodiversity, population genetics at the level of relatedness, genome mapping, as markers for pathogens, etc. The main objective of the present investigation was to study the genetic relationship among wheat genotypes based on SSR markers.

\section{MATERIALS AND METHODS}

Materials: A total of 73 genotypes including 65 hexaploid (Triticum aestivum L.) and 8 tetraploid (Triticum durum $\mathrm{L}$ ) wheat varieties were used in this study (Table 1).

\section{A-DNA extraction}

Genomic DNA was extracted from young leaves of 2-weeks-old seedlings, bulked from 5 different plants per genotype following the CTAB procedure described by Murray and Thompson (1980) with some modifications. The quantity and quality of genomic DNA was tested by the spectrophotometer and agarose gel electrophoresis.

\section{B-Microsatellite markers analysis:}

Eleven wheat microsatellite markers for eleven loci representing at least one microsatellite marker from chromosomes (2A, 3A, 3B, 3D, 4A, 4B, 4D, 5D, 7A, 7B and 7D) (Table 2) were selected for genotyping Röder et al. (1998). The primer sequence of Xtaglgap was described by Devos et al. (1995). All Gatersleben Wheat Microsatellites (Xgwm) used were dinucleotide repeats, whereas Taglgap has a trinucleotide motif. Microsatellite amplifications were carried out as reported by Röder et al. (1998). Polymerase chain reaction and fragment analysis were performed according to Devos et al. (1995) and Röder et al. (1998) (Table 2). Fragment detection for SSR markers was carried out as given in Röder et al. (1998). GWM designation, chromosomal location, motif and fragment size location in 'CS' (bp) of the amplified loci were reported by Röder et al. (1998).

Each PCR was carried out in a about $25 \mu \mathrm{l}$ reaction volume containing double distilled deionizer $\mathrm{H}_{2} \mathrm{O}$, 10x buffer, $\mathrm{MgCl}_{2}$, dNTPs, Taq polymerase, and both primer pairs according to the primers profile. The PCR amplification of wheat genomic DNA was done by incubating the DNA samples for 5 minutes at $94^{\circ} \mathrm{C}$, then 45 cycles comprising $94^{\circ} \mathrm{C}$ for 60 seconds, annealing of primer for 60 seconds at $58-60^{\circ} \mathrm{C}$ and the extension for 60 seconds at $72^{\circ} \mathrm{C}$. The final extension was carried out for 10 minutes at $72^{\circ} \mathrm{C}$. The PCR products were electrophorized on $2.5 \%$ of agrose gel containing $8 \mu \mathrm{l}$ ethidium bromide, at 80 volts for 3-3.5 hours and observed under a UV transilluminator.

\section{Band scoring and data analysis}


Banding pattern of the SSR markers in the individuals were scored as presence (1) and absence (0) of the band. The pairwise comparisons between the tested genotypes were used to calculate the coefficient of genetic similarity matrix (Gs) according to Nei and $\mathrm{Li}$ (1979). The similarity matrix was subjected to cluster analysis using un weighted pair group method with arithmetic means (UPGMA) clustering procedure and a dendrogram was generated using the software package MVSP (Multi-Variate Statistical Package).

To evaluate the efficiency of selected primers for investigation of genetic diversity, the polymorphism information content (PIC) was calculated as: $\left[\mathrm{PIC}=1-\Sigma f^{2}\right]$ where, $f i$ is the frequency of the $i^{\text {th }}$ allele (Weir 1990). The Resolving power (Rp) of each primer was calculated according to Prevost and Wilkinson (1999) as: $\mathrm{Rp}=\Sigma \mathrm{lb}$ where, $\mathrm{lb}=$ band informativeness, $\mathrm{lb}=1-[2 \times(0.5-p)]$ and $p=$ total number of bands present.

\section{RESULTS AND DISCUSSION}

Microsatellites combine many desirable marker properties including abundant, high levels of polymorphism and information content (PIC), high reproducibility, codominance, rapid and simple genotyping assays, uniform genome coverage, and specific polymerase chain reaction (PCR) based assays (Röder et al., 1998). Furthermore, the analysis of microsatellites based on PCR is simple to perform. In wheat, simple sequence repeats (SSR) have been successfully used in a wide range of applications such as genotype identification (Prasad et al., 2000; Zeb et al., 2009 and Salem and Mattar, 2014), diversity studies (Bo"rner et al., 2000; Huang et al., 2002; Zeb et al., 2009; Salem et al., 2008; Akfirat and Uncuoglu 2013; Bousba et al., 2013; Amir et al., 2014 and Khavarinejad, 2014) and quantitative trait loci (QTL) analysis (Bo"rner et al., 2002 and Salem et al., 2007).

Eleven microsatellite markers were used to test the genetic diversity of seventy-three of wheat genotypes $(65$ bread wheat and 8 durum wheat) (Table 2 and Figs. 1, 2). All microsatellite markers allowed identifying DNA fragments amplified polymorphic patterns from genomic extracts of wheat genotypes, yielding a polymorphism. The total number of detected alleles was 89 with an average allele number of 8.1 per locus. The number of alleles per locus ranged from one for the SSR-11 locus to 14 for the SSR-1 locus (Table 2). Their fragment size ranged from 1007 bp in SSR-7 to 93 bp in SSR-10. Different numbers of SSR alleles have been detected in wheat using microsatellite markers. Prasad et al. (2000) found 7.4 averages allele numbers in 55 elite of wheat genotypes. Röder et al. (2002) detected an average of 10.5 alleles per marker from 19 wheat microsatellites in 502 European wheat varieties. Whereas, Huang et al. (2002) used 26 microsatellites to investigate 998 Genbank accessions of wheat originating from different 68 countries and thereby recorded an average allele number of 18.1. Khlestkina et al. (2004) detected average allele numbers of 6.6 in 54 common spring wheat varieties. Akfirat and Uncuoglu (2013) found an average allele number of 3.9 in seven Turkish winter bread wheat genotypes.

The genotype (G29) displayed the highest number of alleles (77 alleles) followed by G28, G49 and G52 (76 alleles, each), while G6 (49 alleles) followed by $\mathrm{G} 4$ ( 50 alleles) revealed the least number of alleles (Table 3). These variations in number of bands amplified by different primers influenced by variable factors such as primer structure and number of annealing sites in the genome (Kernodle et al., 1993).

The maximum number of polymorphic alleles (9 bands) was obtained by the primer SSR- 9 while the minimum number ( 1 allele) was recorded for primer SSR-11 with an average 5 alleles per primer. Polymorphism percentage ranged from as low as $50 \%$ (SSR- 1 , SSR-2 and SSR-5) to as high as 100\% (SSR-10 and SSR-11) (Table 65). Average polymorphism across all the 73 wheat genotypes was found to be $68.24 \%$. A significant correlation $(0.80$, $\mathrm{p}<0.01)$ was observed between the total 
Table 1: Pedigree of tetraploid and hexaploid wheat genotypes used in the study.

\begin{tabular}{|c|c|c|c|c|c|c|c|c|}
\hline Code & Name & Pedigree & Code & Name & Pedigree & Code & Name & Pedigree \\
\hline G1 & SOHAG 1 & GDOVZ469/JO//61.130/LDS & G26 & Nour & $\begin{array}{l}\text { selected early maturing inbred line (F14) } \\
\text { derived from a cross between } \\
\text { Shenap^Sakha69 }\end{array}$ & G51 & ICR-35 & ICARDA \\
\hline G2 & SOHAG 3 & $\begin{array}{l}\text { MEXICALI/MAGHREBI } \\
\text { 72//51792/DURUM\#6 }\end{array}$ & G27 & $1 \times 15$ & $\begin{array}{c}\text { Advanced breeding line derived from inter } \\
\text { population-interenvironmental cross } \\
\text { between early segregates selected in two } \\
\text { contrasting enviroments }\end{array}$ & G52 & ICR-39 & ICARDA \\
\hline G3 & BANI SEWEF 1 & JO"S"/AA"S"/FG"S" & G28 & Line 6 & $\begin{array}{l}\text { Advanced long spike, short statured } \\
\text { inbred line derived from a cross between } \\
\text { two landraces collected from dry areas in } \\
\text { Upper Egypt (Omara, 1994) }\end{array}$ & G53 & ICR-DH18 & ICARDA \\
\hline G4 & BANI SUEF 5 & DIPPER-2/ BUCHEN-3 & G29 & $\begin{array}{l}\text { L.S.15 (Long } \\
\text { spike 15) }\end{array}$ & $\begin{array}{c}\text { An advanced long-spike inbred line (F14) } \\
\text { derived from a cross among landraces } \\
\text { collected from stress areas in Upper Egypt } \\
\text { (Omara,1994) }\end{array}$ & G54 & ICR-49 & ICARDA \\
\hline G5 & SVEVO & Cimmyt's Line / Zenit & G30 & SIDS 1 & HD2173/PAVON"S"//1158.57/MAYA 74 "S" & G55 & HD-38 & ICARDA \\
\hline G6 & Ciccico & $\begin{array}{l}\text { APPULO/NALNOVA(FG)//VALFORTE/PA } \\
\text { TRIZIO(F5) }\end{array}$ & G31 & SIDS 4 & $\begin{array}{c}\text { MAYA"S"/MON"S"//CMHH7A.592/3/GIZA } \\
157^{\star 2}\end{array}$ & G56 & Madden & Gamenya//Gabo*3/Khapstein \\
\hline G7 & WK-12 & $\begin{array}{l}\text { The landraces were originally collected } \\
\text { from farmers' fields near Dandara } \\
\text { Temple at Qena Governorate in } 1993 \\
\text { (Omara, 1994) and were grown since } \\
\text { then every year in order to ascertain the } \\
\text { stability of the black glume character. }\end{array}$ & G32 & SIDS 12 & $\begin{array}{c}\text { BUC//7C/ALD/5/MAYA74/ON//1160.147/3/B } \\
\text { B/GL4/CHAT"S" } \\
\text { /6/MAYA/VUL//CMH74A.63014*SX }\end{array}$ & G57 & $\begin{array}{l}\text { ALTAISKAYA } \\
50\end{array}$ & Unknown \\
\hline G8 & LOCAL SBW & unknown & G33 & $\begin{array}{l}\text { Lerma Rojo } \\
64\end{array}$ & $\begin{array}{l}\left((\text { Yaqui 50*(Norin 10*Brevor) })^{*} \text { Lerma }\right. \\
52)^{*}(\text { Lerma Rojo) } 2\end{array}$ & G58 & $\begin{array}{c}\text { HONGMANGM } \\
\mathrm{Al}\end{array}$ & OROFENIDIZIUZHAO \\
\hline G9 & GIZA 157 & GIZA155//PIT62/LR64/3/TZPP/KNOTT & G34 & TOBARI-66 & $\begin{array}{c}\text { TEZANOS-PINTOS-PRECOZ/SONORA-64- } \\
\text { A[114][144][145][39][1765]; }\end{array}$ & G59 & SAHEL 1 & NS732/PIMA/VEE\#5 \\
\hline G10 & GIZA 160 & CHENAB70/GIZA155 & G35 & $\begin{array}{c}\text { SALAMBO } \\
80\end{array}$ & PATO/ICCINIA & G60 & Ejaseed-3 & Unknown \\
\hline G11 & GIZA 163 & F61.70/BON//CNO67/7C & G36 & $\begin{array}{l}\text { BACANORA } \\
\text { T } 88\end{array}$ & JUPIBJY/IURES & G61 & CONDOR & WW15*2/3/PJ/GB56//TZPP/NA160 \\
\hline G12 & GIZA 164 & KVZ/BUHO//KAL/BB & G37 & Sonora 64 & $\begin{array}{c}\text { YAKTANA-5//NORIN- } \\
\text { 10/BREVOR/3/2²YAQUII-54 } \\
\end{array}$ & G62 & $\begin{array}{c}\text { MIRONOVCHA } \\
\text { NKA }\end{array}$ & WEIHENSTEPHEN M./MIRONOV-YA 808 \\
\hline G13 & GIZA 165 & CIANO F 67/MARIS FUNDIN/MONCHO & G38 & DEBEIRA & $\begin{array}{c}\mathrm{HD2160/5/TOB/CNO67//BB/3/NA160*2//TT/S} \\
\text { N64/4/HD1954 }\end{array}$ & G63 & NASMA & $\begin{array}{c}\text { BT1149/BT2511 or } \\
\text { BT1149//Florence/Aurore C }\end{array}$ \\
\hline G14 & GIZA 168 & MIL/BUC//SERI & G39 & EL NIELAIN & S948.A1/7×SANTA ELENA & G64 & GOUMRIA-15 & ICW94-0029-0L-6AP-3AP-2AP-0APS-0AP \\
\hline G15 & SAKHA 8 & CNO67//SN64/KLRE/3/8156 & G40 & MEXIPAK65 & PENJAMO62/GABO55 & G65 & Arrehane & L222 (KLDN) \\
\hline G16 & SAKHA 69 & INIA/RL4220//7C/3/YR & G41 & PAVON F 76 & VCM//CNO/7C/3/KAL/BB & G66 & Saada & BUTTE/BUTTE/ARTHUR71 \\
\hline G17 & SAKHA 92 & NAPO/INIA/WREN & G42 & KBG-01 & 300-SM-501-M/HAR-1709 & G67 & Marchouch & KAL/CNO//28156/3/BT908 \\
\hline G18 & SAKHA 93 & SAKHA 92/TR 810328 & G43 & CHAM 4 & FLK/HORK & G68 & HUBARA-5 & Unknown \\
\hline G19 & SAKHA 94 & OPATA/RAYON/3/JUP/BJYI/URES & G44 & CHAM 6 & W3918A/JUP & G69 & BerKum & Unknown \\
\hline G20 & GEMMIZA 7 & CMH74.630/5X/SERI82/3/AGENT & G45 & GHAM-8 & ICARDA & G70 & Canada-462 & Unknown \\
\hline G21 & GEMMIZA 9 & ALD'S'/HUAC'S'//CMH74.630/5X & G46 & Reyne 28 & ICARDA ( CIMMYT breeding line) & G71 & Canada-515 & Unknown \\
\hline G22 & Gemmieza 10 & Maya 74 & G47 & Aguilal & SAIS*2/KS85241-14 & G72 & GURAB-2 & ICARDA \\
\hline G23 & Shandaweel1 & Site//Mo/4/Nac/Th.Ac./3*Pvn///Mirlo/Buc & G48 & ATTILA & ND/VG9144//KAL/BB/3/YACO/4/VEE\#5 & G73 & GK BENCE & ATR/SAVA/LIB \\
\hline G24 & Misr 1 & OASIS/SKAUZ//4*BCN/3/2*PASTOR & G49 & ICR-DH & ICARDA & & & \\
\hline G25 & $\begin{array}{l}\text { US3-2 (LIRA } \\
\text { SA 92) }\end{array}$ & KVZ/TRM//PTM/ANA & G50 & ICR-33 & ICARDA & & & \\
\hline
\end{tabular}

Table 2. The codes and sequence of SSR primers used for amplification with the number of Total bands (TB), polymorphic bands (PB), percentage of polymorphism (PP), polymorphism information content (PIC) and resolving power (RP) for each primer.

\begin{tabular}{|c|c|c|c|c|c|c|c|}
\hline \multicolumn{2}{|c|}{ Primer code } & Primer sequence & TB & PB & $\mathbf{P P}$ & PIC & $\mathbf{R P}$ \\
\hline \multirow{2}{*}{ 1-Xgwm160 } & SSR-F & "5'-GAATTTACAAACTTCCTTGAGC-3' & \multirow{2}{*}{14} & \multirow{2}{*}{7} & \multirow{2}{*}{50} & \multirow{2}{*}{0.15} & \multirow{2}{*}{2.79} \\
\hline & SSR-R & 5'-AAAAATATACAGCAGTAATCATCTG-3' & & & & & \\
\hline \multirow{2}{*}{ 2-Xtaglgap } & SSR-F & 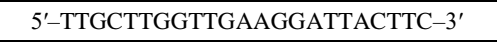 & \multirow{2}{*}{8} & \multirow{2}{*}{4} & \multirow{2}{*}{50} & \multirow{2}{*}{0.07} & \multirow{2}{*}{0.63} \\
\hline & SSR-R & 5'-CCCTCGTAGGAGACCTTCTTT-3' & & & & & \\
\hline \multirow{2}{*}{ 3-Xgwm577 } & SSR-F & 5'-GGGAGGCTGAGGGAATTGTC-3' & \multirow{2}{*}{4} & \multirow{2}{*}{3} & \multirow{2}{*}{75} & \multirow{2}{*}{0.2} & \multirow{2}{*}{1.04} \\
\hline & SSR-R & 5'-AGTGCCGCTGAATTCAGTGAAA-3' & & & & & \\
\hline \multirow{2}{*}{ 4-Xgwme695 } & SSR-F & 5'-AAGAGGCAGAGATGGAGTTC-3' & \multirow{2}{*}{11} & \multirow{2}{*}{6} & \multirow{2}{*}{54.5} & \multirow{2}{*}{0.10} & \multirow{2}{*}{1.29} \\
\hline & SSR-R & 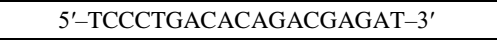 & & & & & \\
\hline \multirow{2}{*}{ 5-Xwmc596 } & SSR-F & 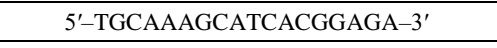 & \multirow{2}{*}{8} & \multirow{2}{*}{4} & \multirow{2}{*}{50} & \multirow{2}{*}{0.07} & \multirow{2}{*}{0.68} \\
\hline & SSR-R & 5'-ATACACGGTGGAAGTTGGC-3' & & & & & \\
\hline \multirow{2}{*}{ 6-Xgwm497 } & SSR-F & 5'-GTAGTGAAGACAAGGGCATT-3' & \multirow{2}{*}{13} & 6 & 46.15 & 0.06 & 093 \\
\hline & SSR-R & 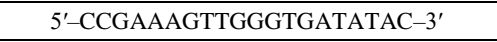 & & $\mathbf{0}$ & 40.15 & 0.00 & 0.93 \\
\hline 7-Xowm 260 & SSR-F & 5'-CACGAAGAGATATCACCCCGAG-3' & 9 & 7 & 7778 & 016 & 107 \\
\hline - $-\mathrm{Ag} \mathrm{gWm} 200$ & SSR-R & 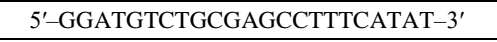 & 9 & 1 & 11.10 & 0.10 & 1.91 \\
\hline & SSR-F & $\begin{array}{l}5^{\prime}-\text { TTTCTTCCGCATCAAGAGATCC-3' } \\
\end{array}$ & 7 & 4 & 57.14 & 0.12 & 1.04 \\
\hline 8-XgwmI/4 & SSR-R & $\begin{array}{l}5^{\prime}-\mathrm{CCTCAGGCTATGGCACAGAAT-3'} \\
\end{array}$ & 1 & 4 & $5 / .14$ & 0.12 & 1.04 \\
\hline 9-Xowm635 & SSR-F & 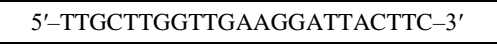 & 10 & 9 & 90 & 0.14 & 1.64 \\
\hline & SSR-R & 5'-CCCTCGTAGGAGACCTTCTTT-3' & 10 & Y & 80 & 0.14 & 1.04 \\
\hline 10-Xowm 573 & SSR-F & 5'-GGGAGGCTGAGGGAATTGTC-3' & 4 & 4 & 100 & 014 & 0.66 \\
\hline 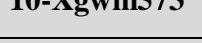 & SSR-R & 5'-AGTGCCGCTGAATTCAGTGAAA-3' & 4 & 4 & 100 & 0.14 & 0.00 \\
\hline 11-Xgwmc182 & SSR-F & 5'-AAGAGGCAGAGATGGAGTTC-3' & 1 & 1 & 100 & 0.20 & 0.22 \\
\hline 11-Xgwmeis & SSR-1R & 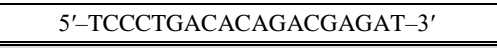 & 1 & 1 & 100 & 0.20 & 0.22 \\
\hline Average & & & 8.09 & 5.00 & 68.24 & 0.13 & 1.17 \\
\hline
\end{tabular}


Table (3): Number of amplified DNA-fragments in seventy-three wheat genotypes investigated with eleven SSR primers.

\begin{tabular}{|c|c|c|c|c|c|c|c|c|c|c|c|c|c|c|c|c|c|c|c|c|c|c|c|c|c|}
\hline 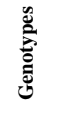 & $\overline{\vec{v}}$ & $\begin{array}{l}\tilde{\tilde{u}} \\
\tilde{w}\end{array}$ & 岂 & $\underset{\overrightarrow{\tilde{v}}}{\overrightarrow{\tilde{w}}}$ & $\begin{array}{l}\mathscr{\tilde { z }} \\
\tilde{z}\end{array}$ & : & 它 & \begin{tabular}{l} 
o \\
\multirow{u}{*}{}
\end{tabular} & 容 & $\begin{array}{l}\stackrel{\ominus}{\vec{j}} \\
\dot{\tilde{u}}\end{array}$ & $\begin{array}{l}\overline{7} \\
\dot{\tilde{n}}\end{array}$ & & 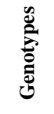 & $\begin{array}{l}\overrightarrow{\tilde{u}} \\
\bar{n}\end{array}$ & $\begin{array}{l}\tilde{\tilde{u}} \\
\tilde{\tilde{w}}\end{array}$ & $\begin{array}{l}\vec{z} \\
\tilde{n} \\
\frac{n}{n}\end{array}$ & 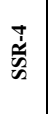 & $\begin{array}{l}\stackrel{w}{\tilde{z}} \\
\tilde{w}\end{array}$ & 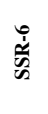 & 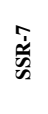 & 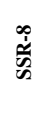 & $\frac{\hat{\tilde{u}}}{\tilde{w}}$ & 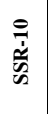 & $\begin{array}{l}\overline{\vec{\nu}} \\
\dot{\tilde{u}}\end{array}$ & స్ \\
\hline G1 & 7 & 6 & 2 & 7 & 7 & 9 & 5 & 4 & 4 & 0 & 0 & 51 & G38 & 14 & 7 & 4 & 11 & 7 & 8 & 4 & 6 & 7 & 2 & 1 & 71 \\
\hline G2 & 7 & 6 & 2 & 7 & 7 & 9 & 7 & 4 & 4 & 0 & 0 & 53 & G39 & 13 & 7 & 4 & 11 & 8 & 9 & 4 & 7 & 8 & 2 & 1 & 74 \\
\hline G3 & 7 & 6 & 2 & 7 & 7 & 9 & 5 & 4 & 4 & 0 & 0 & 51 & G40 & 13 & 7 & 3 & 11 & 7 & 8 & 5 & 6 & 8 & 2 & 1 & 71 \\
\hline G4 & 7 & 6 & 2 & 7 & 7 & 9 & 4 & 4 & 4 & 0 & 0 & 50 & G41 & 13 & 7 & 3 & 11 & 8 & 9 & 5 & 7 & 7 & 2 & 1 & 73 \\
\hline G5 & 7 & 6 & 2 & 7 & 8 & 10 & 5 & 5 & 4 & 0 & 0 & 54 & G42 & 13 & 7 & 3 & 11 & 7 & 8 & 5 & 6 & 7 & 2 & 1 & 70 \\
\hline G6 & 7 & 6 & 2 & 7 & 5 & 9 & 5 & 4 & 4 & 0 & 0 & 49 & G43 & 12 & 7 & 4 & 10 & 8 & 9 & 4 & 7 & 8 & 2 & 1 & 72 \\
\hline G7 & 7 & 7 & 2 & 7 & 7 & 9 & 6 & 4 & 4 & 0 & 0 & 53 & G44 & 12 & 7 & 4 & 10 & 8 & 9 & 4 & 7 & 8 & 2 & 1 & 72 \\
\hline G8 & 7 & 6 & 2 & 7 & 8 & 10 & 6 & 5 & 4 & 0 & 0 & 55 & G45 & 12 & 7 & 4 & 10 & 7 & 8 & 3 & 6 & 8 & 2 & 1 & 68 \\
\hline G9 & 12 & 7 & 3 & 11 & 7 & 9 & 4 & 6 & 8 & 2 & 1 & 70 & G46 & 11 & 7 & 3 & 11 & 7 & 8 & 4 & 6 & 8 & 2 & 1 & 68 \\
\hline G10 & 12 & 7 & 3 & 11 & 7 & 8 & 5 & 6 & 8 & 2 & 1 & 70 & G47 & 11 & 7 & 3 & 11 & 7 & 8 & 5 & 6 & 8 & 2 & 1 & 69 \\
\hline G11 & 12 & 7 & 3 & 11 & 7 & 8 & 5 & 6 & 8 & 2 & 1 & 70 & G48 & 12 & 7 & 3 & 11 & 7 & 8 & 6 & 6 & 8 & 2 & 1 & 71 \\
\hline G12 & 12 & 7 & 3 & 11 & 7 & 8 & 5 & 6 & 8 & 2 & 1 & 70 & G49 & 12 & 8 & 3 & 11 & 8 & 9 & 5 & 7 & 9 & 3 & 1 & 76 \\
\hline G13 & 13 & 7 & 3 & 11 & 7 & 8 & 5 & 6 & 8 & 2 & 1 & 71 & G50 & 12 & 7 & 2 & 11 & 7 & 8 & 5 & 6 & 9 & 2 & 1 & 70 \\
\hline G14 & 13 & 7 & 3 & 11 & 8 & 9 & 4 & 7 & 8 & 2 & 1 & 73 & G51 & 12 & 7 & 2 & 10 & 7 & 8 & 5 & 6 & 9 & 2 & 1 & 69 \\
\hline G15 & 11 & 7 & 3 & 11 & 7 & 8 & 4 & 6 & 7 & 2 & 1 & 67 & G52 & 12 & 7 & 4 & 11 & 8 & 9 & 6 & 7 & 9 & 2 & 1 & 76 \\
\hline G16 & 11 & 7 & 3 & 11 & 7 & 8 & 4 & 6 & 7 & 2 & 1 & 67 & G53 & 12 & 7 & 3 & 11 & 7 & 8 & 5 & 6 & 9 & 2 & 1 & 71 \\
\hline G17 & 11 & 7 & 3 & 11 & 7 & 8 & 5 & 6 & 6 & 2 & 1 & 67 & G54 & 12 & 8 & 2 & 11 & 7 & 8 & 4 & 6 & 9 & 2 & 1 & 70 \\
\hline G18 & 11 & 7 & 3 & 11 & 7 & 8 & 4 & 6 & 7 & 2 & 1 & 67 & G55 & 12 & 7 & 4 & 11 & 7 & 8 & 4 & 6 & 9 & 2 & 1 & 71 \\
\hline G19 & 11 & 7 & 3 & 11 & 8 & 9 & 5 & 7 & 7 & 2 & 1 & 71 & G56 & 12 & 7 & 4 & 11 & 7 & 8 & 4 & 6 & 8 & 2 & 1 & 70 \\
\hline G20 & 9 & 7 & 3 & 11 & 7 & 9 & 5 & 6 & 8 & 1 & 1 & 67 & G57 & 13 & 8 & 2 & 10 & 8 & 9 & 5 & 6 & 7 & 1 & 1 & 70 \\
\hline G21 & 9 & 7 & 3 & 11 & 7 & 9 & 4 & 6 & 8 & 1 & 1 & 66 & G58 & 13 & 7 & 3 & 11 & 8 & 9 & 5 & 7 & 7 & 2 & 1 & 73 \\
\hline G22 & 9 & 5 & 3 & 11 & 7 & 9 & 7 & 6 & 8 & 1 & 1 & 67 & G59 & 14 & 7 & 3 & 11 & 7 & 8 & 5 & 6 & 8 & 2 & 1 & 72 \\
\hline G23 & 12 & 7 & 4 & 11 & 7 & 8 & 4 & 6 & 8 & 2 & 1 & 70 & G60 & 12 & 7 & 4 & 11 & 7 & 8 & 5 & 6 & 8 & 2 & 1 & 71 \\
\hline G24 & 12 & 7 & 4 & 11 & 7 & 8 & 4 & 6 & 8 & 2 & 1 & 70 & G61 & 12 & 7 & 3 & 11 & 7 & 8 & 3 & 6 & 8 & 2 & 1 & 68 \\
\hline G25 & 13 & 8 & 4 & 9 & 7 & 9 & 4 & 7 & 7 & 2 & 1 & 71 & G62 & 13 & 7 & 3 & 10 & 7 & 8 & 4 & 5 & 7 & 1 & 1 & 66 \\
\hline G26 & 13 & 8 & 3 & 9 & 7 & 9 & 4 & 7 & 7 & 2 & 1 & 70 & G63 & 13 & 7 & 3 & 11 & 7 & 8 & 4 & 6 & 8 & 2 & 1 & 70 \\
\hline G27 & 13 & 8 & 4 & 9 & 7 & 9 & 3 & 7 & 7 & 2 & 1 & 70 & G64 & 14 & 7 & 3 & 11 & 7 & 8 & 5 & 6 & 8 & 2 & 1 & 72 \\
\hline G28 & 13 & 8 & 3 & 10 & 8 & 9 & 5 & 7 & 9 & 3 & 1 & 76 & G65 & 14 & 7 & 3 & 11 & 7 & 8 & 5 & 6 & 8 & 2 & 1 & 72 \\
\hline G29 & 13 & 8 & 4 & 10 & 8 & 9 & 5 & 7 & 9 & 3 & 1 & 77 & G66 & 14 & 7 & 2 & 11 & 7 & 8 & 5 & 6 & 8 & 2 & 1 & 71 \\
\hline G30 & 12 & 7 & 4 & 11 & 7 & 8 & 4 & 6 & 8 & 2 & 1 & 70 & G67 & 13 & 7 & 4 & 11 & 7 & 8 & 4 & 6 & 8 & 2 & 1 & 71 \\
\hline G31 & 13 & 7 & 3 & 10 & 7 & 8 & 3 & 6 & 8 & 2 & 1 & 68 & G68 & 12 & 7 & 3 & 11 & 7 & 8 & 4 & 6 & 8 & 2 & 1 & 69 \\
\hline G32 & 12 & 7 & 3 & 11 & 7 & 8 & 3 & 6 & 8 & 2 & 1 & 68 & G69 & 13 & 7 & 2 & 11 & 7 & 8 & 4 & 6 & 8 & 2 & 1 & 69 \\
\hline G33 & 12 & 7 & 3 & 11 & 7 & 8 & 4 & 6 & 8 & 2 & 1 & 69 & G70 & 12 & 7 & 4 & 11 & 7 & 8 & 3 & 6 & 8 & 2 & 1 & 69 \\
\hline G34 & 14 & 7 & 4 & 11 & 7 & 8 & 4 & 6 & 8 & 2 & 1 & 72 & G71 & 12 & 7 & 3 & 11 & 7 & 8 & 3 & 6 & 8 & 2 & 1 & 68 \\
\hline G35 & 14 & 7 & 4 & 11 & 8 & 9 & 4 & 7 & 7 & 2 & 1 & 74 & G72 & 12 & 7 & 3 & 11 & 7 & 8 & 2 & 6 & 7 & 2 & 1 & 66 \\
\hline G36 & 14 & 7 & 4 & 11 & 7 & 8 & 3 & 6 & 7 & 2 & 1 & 70 & G73 & 12 & 7 & 3 & 11 & 8 & 9 & 4 & 7 & 8 & 2 & 1 & 72 \\
\hline \multirow[t]{3}{*}{ G37 } & 12 & 7 & 3 & 11 & 8 & 9 & 4 & 7 & 7 & 2 & 1 & 71 & \multirow{2}{*}{ 吾 } & \multirow[b]{2}{*}{$\ddot{\infty}$} & \multirow[b]{2}{*}{$\frac{\theta}{n}$} & \multirow{2}{*}{ 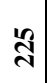 } & \multirow{2}{*}{$\stackrel{7}{12}$} & \multirow{2}{*}{ กั } & \multirow{2}{*}{$\frac{0}{6}$} & \multirow{2}{*}{ लె } & \multirow[b]{2}{*}{ 导 } & \multirow[b]{2}{*}{ if } & \multirow[b]{2}{*}{$\stackrel{\infty}{\mathbb{I}}$} & \multirow[b]{2}{*}{ - } & \multirow{2}{*}{$\stackrel{\circ}{\circ}$} \\
\hline & & & & & & & & & & & & & & & & & & & & & & & & & \\
\hline & & & & & & & & & & & & & 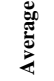 & $\mathcal{I}$ & $r$ & $\vec{m}$ & $\stackrel{\varrho}{\underline{\varepsilon}}$ & $\stackrel{2}{2}$ & $\stackrel{+}{\infty}$ & $\underset{+}{+}$ & $b$ & ra & $\stackrel{\infty}{\rightarrow}$ & $\hat{\theta}$ & $\bar{d}$ \\
\hline
\end{tabular}



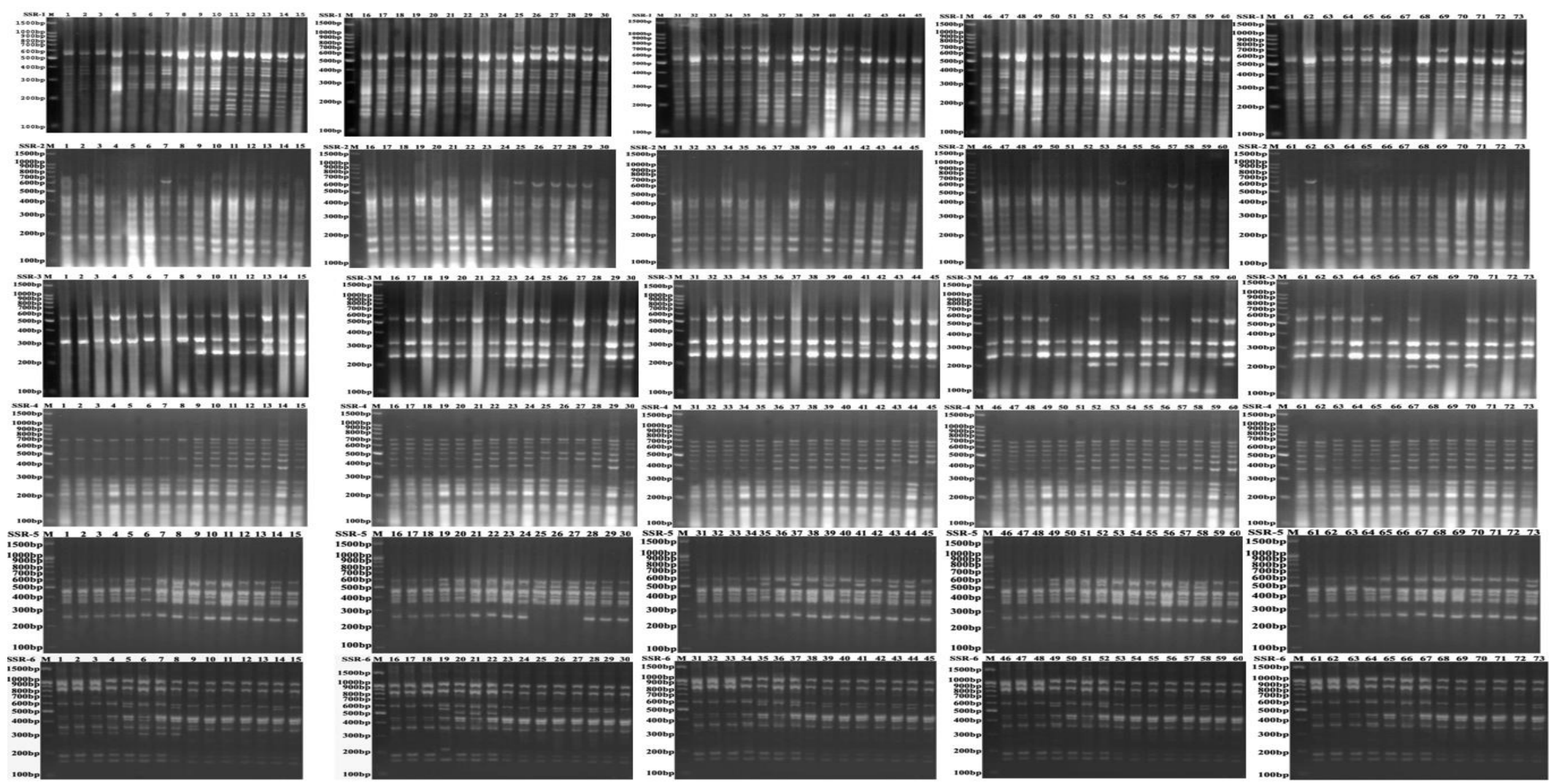

Fig. 1: ISSR banding profile of 8 durum (lanes 1 to 8 ) and 65 bread (lanes 9 to 73) wheat genotypes amplified with the primers SSR1 to SSR 6 . Lane M represented the DNA ladder. 

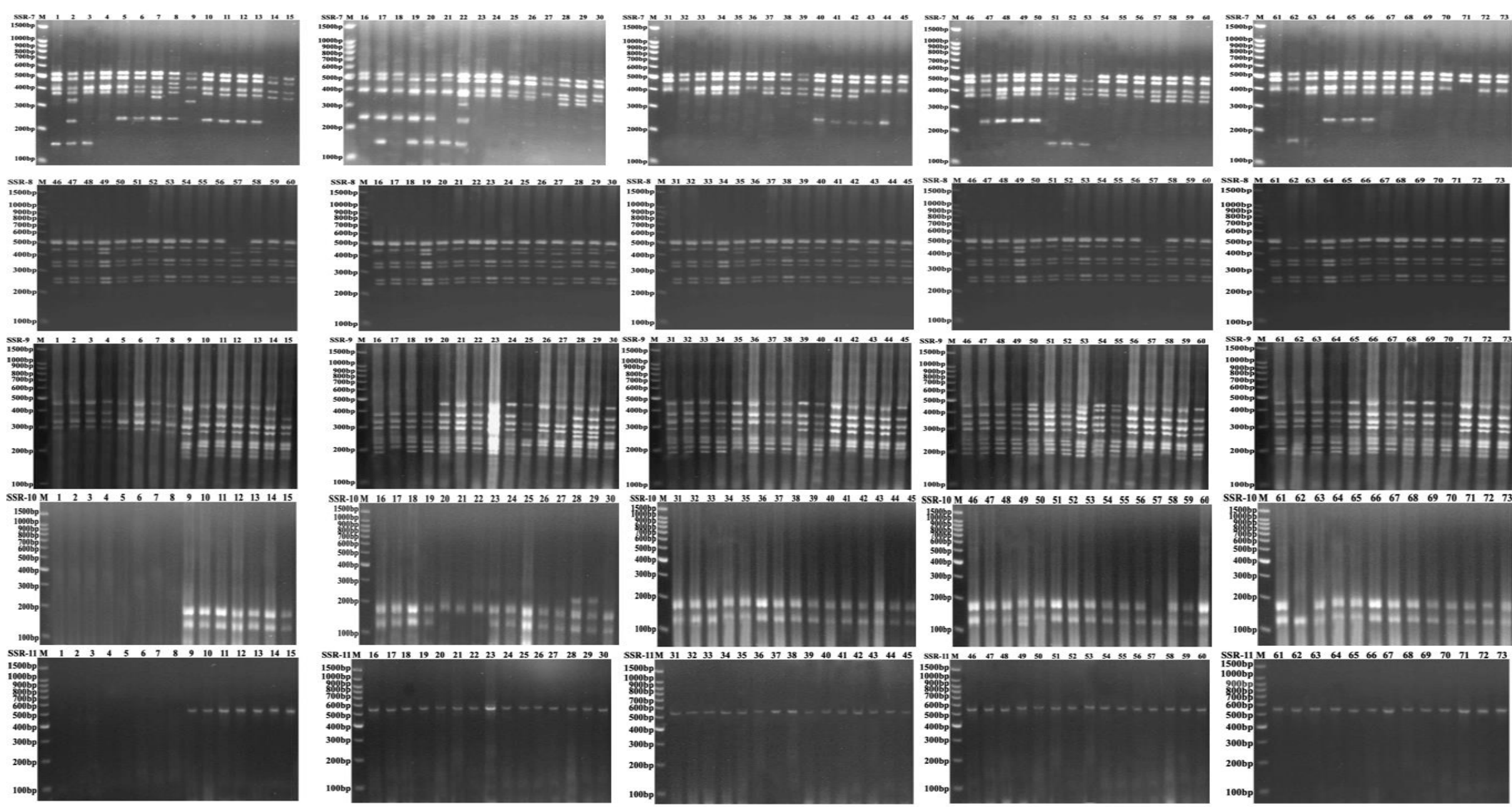

Fig. 1: ISSR banding profile of 8 durum (lanes 1 to 8 ) and 65 bread (lanes 9 to 73 ) wheat genotypes amplified with the primers SSR7 to SSR 11 . Lane $M$ represented the DNA ladder. 


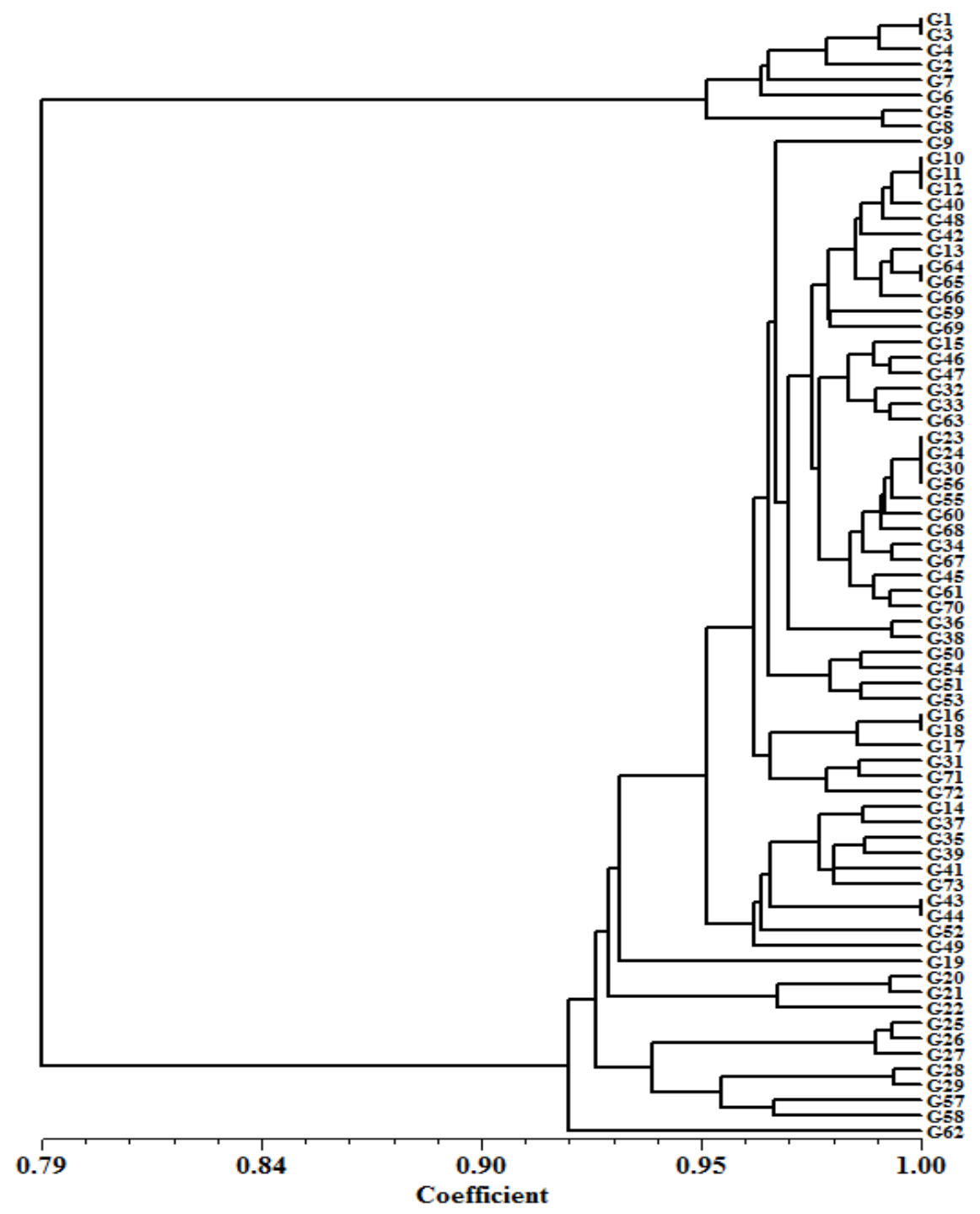

Fig. (3): Dendrogram of 73 wheat genotypes developed from SSR data using UPGMA analysis. The scale is based on Dice coefficients of similarity.

number of bands and the number of polymorphic bands amplified by the eleven SSR primers. The high level of polymorphism is in accordance with the previously published results (Ro“der et al., 1995; Bryan et al., 1997; Islam et al., 2012; Akfirat and Uncuoglu, 2013; Wang et al., 2013; Salem and Mattar, 2014 and Khaled et al., 2015)

The high level of polymorphism in the present study indicative of greater genetic diversity between the tested wheat genotypes, which include 65 bread wheat and 8 durum wheat genotypes, and

confirmed the efficiency of SSR molecular markers in detecting polymorphism among wheat genotypes. It is generally reported that polymorphism between cultivars can arise through nucleotide changes that prevent amplification by introducing a mismatch at one priming site; deletion of a priming site; insertions that render priming site too distant to support amplification and insertions or deletions that change the size of the amplified product (Powell et al., 1996). Polymorphism also considered as a useful selection tool in monitoring alien genome introgression in wheat breeding programs.

Primers differentiating bread wheat and durum wheat genotypes 
Eleven alleles at molecular size [219 bp and $188 \mathrm{bp}$ (SSR-1), $239 \mathrm{bp}$ (SSR-3), 635 bp and 503 bp (SSR-4), 358 bp and 331 bp (SSR-8), $250 \mathrm{bp}, 226 \mathrm{bp}$ and $210 \mathrm{bp}$ (SSR-9) and $532 \mathrm{bp}$ (SSR-11)], were present only in bread wheat genotypes, while one allele at molecular size 312 bp generated with primer (SSR-6) was unique to durum wheat genotypes. These alleles could be used as marker to distinguish the durum wheat from the bread wheat genotypes.

\section{Genotype Specific Primers}

Eleven SSR primers produced a total of 9 unique bands. There were 9 unique fragments $(10.11 \%)$ that could distinguish genotypes. Maximum number of unique bands (3) was scored with the primer SSR-6. Out of the 73 wheat genotypes, only seven varieties (G22, G6, G9, G19, G8, G72 and G49) possessed unique bands. These unique alleles were; two in G19 [one positive at $215 \mathrm{bp}$ and one negative at $189 \mathrm{bp} \mathrm{(SSR-6)],} \mathrm{two} \mathrm{negative}$ alleles at 439 bp and 389 bp (SSR-2) in G22, one negative allele at $361 \mathrm{bp}$ (SSR-5) in G6, one positive marker at $363 \mathrm{bp}$ (SSR-6) in G9, one positive and one negative alleles with 435 bp and 403 bp (SSR-7) in G8 and G72, respectively, and one positive allele at $93 \mathrm{bp}$ (SSR-10) in G49. Unique SSR alleles have also been reported by Akfirat and Uncuoglu (2013).

\section{Polymorphism information content (PIC)}

The PIC values obtained here are typical of dominant markers in which only two alleles (i.e. states) - presence (1) and absence (0) were assumed for a particular locus (i.e. band or row) and thus by definition the highest frequency for a locus is 0.5 . The PIC value, a parameter indicative of the degree of informativeness of a marker, in this study, the PIC ranged from 0.06 (primer SSR-6) to 0.2 (primer SSR-11) with an average value of 0.13 (Table 2). These results reflect the wide range between the frequencies of alleles at loci of SSR studied in the tested wheat varieties.

Achtara et al. (2010) reported that the PIC values in 49 wheat varieties (37 durum and 12 bread wheat) ranged between 0.0-0.88. However, Bryan et al. (1997) found that PIC value with an average 0.51 from 49 SSR primer pairs isolated from hexaploid wheat genome. The genetic differentiation of 60 wheat cultivars selected for adaptation and end-use from Hungary, Austria, and German using 42 microsatellite showed, on average, PIC value of 0.57 (Stachel et al., 2000).

There was significant correlation between PIC and the number of alleles $(r=0.52, p<0.01)$. Therefore, the number of alleles can be used for the evaluation of genetic diversity. The results obtained in the present investigation were in agreement with those of (Huang et al., 2002; Achtara et al., 2010; Wang et al., 2013; Khavarinejad, 2014 and Reza et al., 2015) who reported that the PIC value was correlated with the number of alleles.

\section{Resolving power (RP)}

The resolving power ( $R p)$ is another method used to measure the ability of primers or techniques to distinguish between genotypes (Prevost and Wilkinson, 1999). The estimates of RP ranged from 0.22 in SSR-11 to 2.79 in SSR-1 with an average of 1.17 per primer (Table 2). Prevost and Wilkinson (1999) reported that the RP index provides a moderately accurate estimate of the number of genotypes identified by a primer. RP was significantly associated with the other molecular indices e.g. PPB and PIC ( $r=0.23$ and $r=0.19$, respectively) in this study. The resolving power provides information on the ability of a primer, i.e. SSR-1 (PR = 2.79), to reflect the genetic or taxonomic relationships of a group of genotypes under study.

\section{Genetic similarity (GS)}

The genetic similarity (GS) for pairs of the genotypes was calculated using the software NTSYS program according of Dice coefficients. The bivariate $(1 / 0)$ data and Dice similarity coefficient matrix of the 73 wheat genotypes, based on the data of eleven SSR primers, were used to construct the dendrogram (Fig. 3).

The similarity indices based on all possible pairs of genotypes ranged from 0.73 to 1.00 with an average 0.87 . Close similarity was observed between G1/G3, G10/G11/G12, G64/G65, G23/G24/G30/G56, G16/G18 and G43/G44 indicating that these genotypes were 
similar to each other at the DNA loci tested by the eleven SSR primers.

The other genotypes displayed moderate to high genetic similarity between them (GS $\leq$ 0.73) which reflects low degree of genetic variability among these genotypes at the tested loci. Several authors have reported a large genetic similarity in wheat (Sehgal et al., 2012 and Wang et al., 2013)

The dendrogram (Fig. 3) grouped the 73 wheat genotypes according to their ploidy levels into two main clusters within a close-off $0.79 \mathrm{GS}$. Cluster 1 included the durum wheat genotypes, while cluster 2 contained all bread wheat genotypes.

The dendrogram also reflected the differences in genetic diversity within the two ploidy levels and discriminated all genotypes. In cluster 1 , the durum wheat genotypes were divided at 0.95 GS into two sub-clusters, the first contains the genotypes (G1, G2, G3, G4, G6 and G7) and the second included G5 and G8.

The bread wheat genotypes were clustered together in several sub-clusters within high similarity levels (GS $\leq 0.92$ ) reflecting low variability between them at the tested SSR loci.

Powell et al. (1996) reported that several factors might affect the estimates of genetic relationships between individuals i.e., number of markers used, distribution of markers in the genome (genome coverage) and the nature of evolutionary mechanisms underlying the variation measured.

The above discussion amply demonstrates the utility of microsatellites, which can be profitably utilized in wheat not only for detecting polymorphism and tagging genes (Prasad et al., 1999 and Salem and Mattar, 2014) but also for genotype identifica-tion and for estimation of genetic diversity.

These results are in agreement with those obtained by (Ro“der et al., 1998; Pestsova et al., 2000; Achtara et al., 2010; Islam et al., 2012; Spanic et al., 2012; Bousba et al., 2013; Wang et al., 2013; Amir et al., 2014 and Khavarinejad, 2014).

REFERENCES
Achtara, S.; M. Y. Mouallaa; A. Kalhoutb; M. S. Röderc and N. Miralid (2010). Assessment of genetic diversity among Syrian durum (Triticum ssp. durum) and bread wheat (Triticum aestivum L.) using SSR markers.

Akfirat, F. S. and A. A. Uncuoglu (2013). Genetic diversity of winter wheat (Triticum aestivum L.) revealed by SSR markers. Biochem Genet., 51: 223-229.

Amir, R.; N. M. Minhas; A. Gul Kazi; S. Farrakh; A. Ali; H. Bux and M. Kazi (2014). Phenotypic and genotypic characterization of wheat landraces of Pakistan. Emirates Journal of Food and Agriculture, 26(2): 157-163.

Borner, A.; E. Schumann; A. Furste; H. Coster and B. Leithold (2002). Mapping of quantitative trait loci determining agronomic important characters in hexaploid wheat (Triticum aestivum L.). Theor Appl Genet., 105: 921-936.

Borner, A.; S. Chebotar and V. Korzun (2000). Molecular characterization of the genetic integrity of wheat (Triticum aestivum L.) germplasm after long-term maintenance. Theor Appl Genet., 100: 494-497.

Bousba, R.; M. Baum; A. jighly; A. Djekoune; S. Lababidi; A. Benbelkacem; $M$. Labhilili; F. Gaboun and N. Ykhlef (2013). Association analysis of genotypic and phenotypic traits using SSR marker in durum wheat. online international interdisciplinary research journal, 3(4): 60-79.

Bryan, G. J.; A. J. Collins; P. Stephensons and A. Orry (1997). Isolation and characterization of microsatellites from hexaploid bread wheat. Theor Appl Genet., 94: 557-563.

Devos, K. M.; G. J. Bryan; A. J. Collins and P. Stephenson (1995) Application of two microsatellite sequences in wheat storage proteins as molecular markers. Theor Appl Genet., 90: 247-252.

Fufa, H.; P. S. Baenziger; B. S. Beecher; I. Dweikat; R. A. Graybosch and K. M. Eskridge (2005). Comparison of phenotypic and molecular marker-based 
classifications of hard red winter wheat cultivars. Euphytica, 145: 133-146.

Huang, X. Q.; A. Bo“rner, M. S. Ro“der and M. W. Ganal (2002). Assessing genetic diversity of wheat (Triticum aestivum L.) germplasm using microsatellite markers. Theor. Appl. Genet., 105: 699-707.

Islam, S.; M. S. Haque; R. M. Emon; M. M. Islam and S. N. Begum (2012). Molecular characterization of wheat (Triticum aestivum L.) genotypes through SSR markers. Bangladesh J. Agril. Res., 37(3): 389-398.

Kernodle, S. P.; R. E. Cannon and J. G. Scandalios (1993). Concentration of primer and template qualitatively affects product in RAPD-PCR. Biotechniques, 1: 362-364.

Khaled, M.; A. Mahjoub; M. Abassi; A. Albouchi; Z. Ouerghi; N. A. Ben-Brahim, Z. Béjaoui (2015). Morphological and genetic variation in (Aegilops geniculata roth.) from Tunisia. International Journal of Agronomy and Agricultural Research, 6(2): 8-21.

Khavarinejad, M. S. (2014). Comparison of obtained wheat genetic divergence by molecular and morphological analysis using cluster. Scientia Agriculturae, 6(3): 107-113.

Khlestkina, E. K.; M. S. Röder; T. T. Efremova; A. Börner and V. K. Shumny (2004). The genetic diversity of old and modern Siberian varieties of common spring wheat as determined by microsatellite markers. Plant Breed., 123: 122-127.

Kihara, H. (1994). Discovery of the DDanalyzer, one of the ancestors of vulgare wheats. Agric. Hortic., 19: 889-890.

Maric, S.; S. Bolaric; J. Martinic; I. Pejic and V. Kozumplik (2004). Genetic diversity of hexaploid wheat cultivars estimated by RAPD markers, morphological traits and coefficients of percentage. Plant Breed., 123: 366-369.

McFadden, E. S. and E. R. Sears (1964). The origin of Triticum spelta and its freethreshing hexaploid relatives. J. Heredity, 37: 81-89.
Murray, M. G. and W. F. Thompson (1980) Rapid isolation of high molecular weight plant DNA. Nucleic Acids Res., 8: 43214325.

Nei, M. and W. H. Li (1979). Mathematical model for studying genetic variation in terms of restriction endonucleases. Proc. Natl. Acad. Sci. USA., 76: 5269-5273.

Palombi, M. A. and C. Damiano (2002). Comparison between RAPD and SSR molecular markers in detecting genetic variation in kiwifruit (Actinidia deliciosa A. Chev). Plant Cell Rep., 20: 1061-1066.

Pejic, I.; P. Ajmone-Marsan; M. Morgante; V. Kozumplick; P. Castiglioni; G. Taramino and M. Motto (1998). Comparative analysis of genetic similarity among maize inbred lines detected by RFLPs, RAPDs, SSRs and AFLPs. Theoretical and Applied Genetics, 97(8): 1248-1255.

Pestsova, E.; M. W. Ganal and M. S. Ro“der (2000). Isolation and mapping of microsatellite markers specific for the $D$ genome of bread wheat. Genome, 43: 689-697.

Powell, W.; M. Morgante; C. Andre; M. Hanafey; J. Vogel; S. Tingey and A. Rafalski. (1996). The comparison of RFLP, RAPD, AFLP and SSR (microsatellite) markers for germplasm analysis. Molecular Breeding, 2: 225-238.

Prasad, M.; R. K. Varshney; J. K. Roy; H. S. Balyan and P. K. Gupta (2000). The use of microsatellites for detecting DNA polymorphism, genotype identification and genetic diversity in wheat. Theoretical and Applied Genetics, 100: 584-592.

Prasad, P. V. V.; P. Q. Craufurd and R. J. Summerfield (1999). Sensitivity of peanut to timing of heat stress during reproductive development. Crop Sci., 39: 1352-1357.

Prevost, A. and M. J. Wilkinson (1999). A new system of comparing PCR primers applied to ISSR fingerprinting of potato cultivars. Theoretical and Applied Genetics, 98: 107-112. 
Reza, M. D.; N. E. Goodarz and S. Aram (2015). Investigation of genetic diversity of some durum and bread wheat genotypes using SSR markers. Journal of Biodiversity and Environmental Sciences, 6(3): 24-32.

Röder, M. S.; J. Plaschke; S. U. Konig; A. Börner; M. E. Sorrells and S. D. Tanksley (1995). Abundance variability and chromosomal location of microsatellite in wheat. Molecular Genetics and Genomics, 246: 327-333.

Röder, M. S.; V. Korzun; K. Wendehake; J. Plaschke; M. H. Tixier; P. Leroy and M. W. Ganal (1998). A microsatellite map of wheat. Genetics, 149: 2007-2023.

Röder, M. S.; K. Wendehake; V. Korzun; G. Bredemeijer; D. Laborie; L. Bertrand; P. Isaac; S. Rendell; J. Jackson; R. J. Cooke; B. Vosman and M. W. Ganal (2002). Construction and analysis of a microsatellite-based database of European wheat varieties. Theor Appl Genet., 106: 67-73.

Salem, K. F. M. and M. Z. Mattar (2014). Identification of microsatellite alleles for salt tolerance at seedling stage in wheat (Triticum aestivum L.). Life Science Journal, 11(12): 1064-1073.

Salem, K. F. M.; A. M. El-Zanaty and R. M. Esmail (2008). Assessing wheat (Triticum aestivum L.) Genetic diversity using morphological characters and microsatellite markers. World Journal of Agricultural Sciences, 4: 538-544.

Salem, K.; M. Röder and A. Börner (2007). Identification and mapping quantitative trait loci for stem reserve mobilisation in wheat (Triticum aestivum L.). Cereal Research Communications, 35(3): 13671374.

Sehgal, S. A.; R. A. Tahir and M. Nawaz (2012). Molecular characterization of wheat genotypes using SSR markers. Int J Bioautomation, 16: 119-128.
Spanic, V.; H. Buerstmayr and G. Drezner (2012). Assessment of genetic diversity of wheat genotypes using microsatellite markers. Periodicum Biologorum, 114(1): 37-42.

Stachel, M.; T. Lelley; H. Grausgrube and J. Vollmann (2000). Application of microsatellites in wheat (Triticum aestivum L.) for studying genetic differentiation caused by selection for adaptation and use. Theoretical and Applied Genetics, 100: 242-248.

Van-Becelaere, G.; E. L. Lumbbers; A. H. Paterson and P. W. Chee (2005). Pedigree-vs DNA marker-based genetic similarity estimates in cotton. Crop Science, 45(6): 2281-2287.

Wang, J.; M. C. Luo; Z. Chen; F. M. You; Y. Wei; Y. Zheng and J. Dvorak (2013). Aegilops tauschii single nucleotide polymorphisms shed light on the origins of wheat D-genome genetic diversity and pinpoint the geographic origin of hexaploid wheat. New Phytol., 198: 925937.

Weir, B. (1990). Genetic data analysis: Methods for discrete population genetic data. Sinauer Assoc. Sunderland, MA.

Williams, J. G. K.; A. R. Kubelik; K. J. Livak; J. A. Rafalski and S. V. Tingey (1990). DNA polymorphisms amplified by arbitrary primers are useful as genetic markers. Nucleic Acids Res., 18: 6531-6535.

Zeb, B.; I. A. Khan; S. Ali; S. Bacha; S. Mumtaz and Z. A. Swati (2009). Study on genetic diversity in Pakistani wheat varieties using simple sequence repeat (SSR) markers. Afr J Biotechnol., 8: 4016-4019. 